\title{
Editorial
}

\section{REALISM IN LEPROSY CONTROL}

The Director of one of the most efficiently run leprosy control projects in India, reviewing progress over a 17-year period, said recently, "After a few years work by survey, education, and treatment methodology, the decline in incidence comes to a halt, suggesting a new ecological balance between the host and the infective agent. Even though the quantum of (leprosy) infection is reduced, new cases go on appearing in almost equal numbers every year. This static condition may give way if more potent drugs and modified methods of work are discovered" (Nilakanta Rao, 1973a).

This experience is shared by many other workers in India. Indeed, Dr K. C. Das, Senior Administrator in the Government national leprosy control programme, reviewing progress over the country as a whole during the past 25 years, said, "Reduction in incidence can only be expected after 15-20 years, but the quantum of infection is reducing, and new cases are mostly of non-infective type" (Das, 1973).

Leprosy control policy in India is directly in line with that recommended by the Expert Leprosy Committee of the World Health Organization. Clearly, employing present internationally accepted procedures, the road to leprosy control in India is going to be hard, long and expensive. India is not at all unique in this. On the other hand, examples do exist where the same techniques, applied with no greater skill and dedication, have produced a very substantial and continuous decline in leprosy incidence, e.g. in Nigeria (Davey, Ross and Nicholson, 1956; Davey, 1957) and Thailand (Pakdi, Sanayakorn and Seal, 1974). Such examples usually relate to static and well disciplined populations.

Clearly, discouraging factors are operative in some countries which do not apply in the same way in others. As communications improve and population mobility increases, discouraging factors are likely to become more dominant. Experience in both E. Nigeria and India has convinced the writer that these are concentrated far more in the spheres of sociology and economics than in biophysics and public health as generally understood (Davey, 1969). It is not realistic to blame a sustained incidence of clinical leprosy on the prolonged incubation of infections arising before chemotherapy was started. The basic problem confronting us is that all too often patients are not coming forward to take chemotherapy in numbers sufficient to have a substantial effect within reasonable time on the spread of the disease. Many surveys give evidence that early infections are concealed. At established treatment clinics, attendance is all too frequently very low. Nilakanta Rao (1973b) gives an average attendance of lepromatous cases of $44 \%$, non-lepromatous $28 \%$. Our own figures, at a centre 200 miles further south, were similar. Ekambaram (1974) at the ELEF project in Tamil Nadu gives $57 \%$ attendance out of total recorded patients. K. C. Das (1973) 
covering India as a whole states that in June and September 1972 although the percentage of recorded cases actually registered for treatment was 91.2 and 92.2 respectively, the percentage who actually took treatment during a three month period was $39.2 \%$ in June and $36.1 \%$ in September. This problem is shared by other countries. In the Dominican Republic, Herrara (1973) gives $40 \%$ of patients as uncontrolled or lost.

In Tanzania, where $32 \%$ of patients defaulted for 1 year or more, Hertroijs (1973) found that defaulters occurred more in the lower age groups, in unmarried patients, in those with tuberculoid leprosy, in non-deformed and non-reactive patients, in patients with a short history. Most arose in the first year of treatment, and especially among those treated at wayside clinics. Other factors were, the farming season, mobility of population, and lack of confidence in modern treatment.

The lesson to be drawn is very obvious. It is that unless patients feel that their leprosy is a menace to life and health, they will not put themselves out to treat it, and assess their economic and social concerns as of higher priority in daily living. This universal human reaction has serious implications in leprosy control, because it is the patient with almost insignificant early lepromatous leprosy who may be discharging from his nose huge numbers of viable Myco. leprae into the environment. What holds good in Zambia also applies in India, with the added very serious problem that the primary menace of leprosy to personal happiness and well-being is seen less in its potential for physical economic disability than in its effect on social acceptance and stability. The results of this in the concealment of overt leprosy are very serious.

Two fresh factors have recently entered into the situation. First comes convincing evidence from two centres of high reputation, that where patients who do attend for treatment are given tablets of dapsone to use at home, up to about $50 \%$ of them may not be taking their tablets regularly (Low and Pearson, 1974; Ellard, Gammon and Harris, 1974). This is obviously not something new. It exposes the same truth, that the dapsone in which we trust, is not being taken by the numbers of patients we hoped for, nor in the dosage we expect. In dealing with chronic illness we cannot expect enthusiasm to be sustained, but personal forgetfulness and indifference are only two small facets of a much more widespread and serious situation. We may make dapsone freely available on the widest possible scale, but it does not follow that patients are going to take it in a way calculated to lead to the control of leprosy in the forseeable future.

A second factor arises from the work of Godal (1974) and Myrvang, Negassi and Lofgren (1973) which shows that leprosy is more contagious than was earlier thought, and that in endemic areas there exists a reservoir of latent infection wider than usually believed. While, as Browne (1974) has shown, many slight infections may be self healing, the balance in others between latent and overt disease may be fine, and easily disturbed by such things as puberty and parturition. There is more leprosy infection than we think. This discovery makes even more pressing the need to find and apply methods of leprosy control which will be widely acceptable.

In practice the central problem is not an economic one. If patients cannot afford to lose a day's wage in order to attend a treatment clinic, it is up to us to circumvent the problem. If, for the sake of work, patients move from place to place, that in itself should be no deterrent to treatment. Much more important than any economic factor is ignorance, and the stubbornly persistent fear of 
leprosy and its destructive social consequences, which determine personal and community attitudes and behaviour.

Clearly the order of our priorities needs to be changed. The aims of case finding and case holding by the offering of acceptable chemotherapy and care are still fundamental, but the situation calls for antecedent steps which need to be given the highest priority. Four of these are readily identifiable.

(1) First undoubtedly is a new approach to the leprosy education of the whole exposed population in endemic areas, and especially people in authority, administrators, doctors and teachers. Amateur methods are no longer acceptable. The approach must be professional, comprehensive, and utilize the mass media. The Gandhi Memorial Leprosy Foundation is a pioneer in this, and doing work of the utmost importance, including courses for the training of educators at all levels. A similar centre is needed in every country where leprosy presents serious social problems.

(2) Any approach to leprosy control and treatment which singles out leprosy for special attention with a separate organization and staff, except at consultant level, is in many places to be thought of as discredited and out of date. It is now of high priority to integrate leprosy into the general health services at ground level, so that chemotherapy can be offered at primary health centres and dispensaries where patients can attend without drawing attention to themselves. This measure at once removes a major source of discouragement to patients. A corollary to it is that other sources of discouragement need to be studied and identified, and the will of patients to take treatment thereby strengthened.

(3) Prophylaxis by the inoculation of a suitable vaccine must now be regarded in principle as of the highest priority, to be applied along with other preventive inoculations, without singling out leprosy as a special disease. The discovery of such a vaccine should soon become a practical possibility. Pending its development, we can at least make use of $\mathrm{BCG}$ as an interim measure. Chemoprophylaxis may have limited usefulness, but cannot be generally recommended, because once again it introduces the personal decision to take or not to take the tablet offered.

(4) Finally, there needs to be more dynamism and flexibility, accepting that special situations may call for ways of approach elsewhere unacceptable. Thus in India, there are large numbers of patients who possess literally nothing, whose ties with their village homes have been permanently broken, and who swell the slums in large cities. Orthodox attitudes to leprosy control would frown on the idea of gathering such people together and resettling them into resident, supervised communities outside cities, where they have land for agriculture, but in fact this is a practical and effective solution to a most intractable problem, in the face of which doctrinaire ideas become irrelevant. Sharma (1973) has described a well organized example of this approach.

The need for changing priorities is well expressed in a recent World Health Organization publication. "Health services are too often tied down by definitions of 'environmental health factors' which underline biological and physical factors as opposed to social and economic aspects; the latter entailing changes in human relationships. The conventional structure of many health services at all levels, be they national, regional, or local, are still geared to deal almost exclusively with biophysical hazards and nuisances. Little time has been found up to now to deal with psycho-social and psycho-economic factors which influence the life and health of people" (Levi, 1974).*

* Italics mine. T.F.D. 
So it is with the leprosy patient. When planning his welfare, it is all too easy to think of him as the pawn in the game, who will fit into a pattern of play without question. He is in fact a person, who will make his own choices whatever we plan, and it behoves us to study his real situation, and devise ways of helping him which preserve his personal dignity and relationships, at the same time as attacking in the community the infection from which he is suffering.

\section{References}

Browne, S. G. (1974). Lepr. Rev. 45, 106.

Das, K. C. (1973). Paper read at the All India Leprosy Workers' Silver Jubilee Conference, October 1973.

Davey, T. F., Ross, C. M. and Nicholson, B. (1956). Brit. med. J. ii, 65.

Davey, T. F. (1957). Int. J. Lepr. 25, 329

Davey, T.F.(1969). Lepr. Rev. 40, 197.

Ekambaram, K. (1974). ELEP Leprosy Control Project, Dharmapuri, 6th Annual Report.

Ellard, G. A., Gammon, P. T. and Harris, J. M. (1974). Lepr. Rev. 45, 224.

Godal, T. (1974). Lepr. Rev. 45, 22.

Herrara, G. (1973). 10th International Leprosy Congress, Abstracts, No. 241, p. 146.

Hertroijs, A. R. (1973). 10th International Leprosy Congress, A bstracts, No. 21 3, p. 128.

Levi (1974). W.H.O. Features May 1974. No. 30.

Low, S. J. M. and Pearson, J. M. R. (1974). Lepr. Rev. 45, 218.

Myrvang, M., Negassi, K. and L $\phi$ fgren, M. (1973). 10th International Leprosy Congress, Abstracts, No. 186, p. 112.

Nilakanta Rao, M. S. (1973a). Paper read at the All India Leprosy Workers Silver Jubilee Conference, October 1973.

Nilakanta Rao, M. S. (1973b). Gandhi Memorial Leprosy Foundation Report for 1972-3, p. 22. Pakdi, A. C., Sanayakorn, C. K. and Seal, K. S. (1974). Lepr. Rev. 45, 205.

Sharma, K. S. (1973). Paper read at the All India Leprosy Workers' Silver Jubilee Conference, October 1973 .

T. F. Davey

\section{Erratum}

A serious misprint occurs in Dr Rees's Editorial "Growing Points in Leprosy Research", on page 2, line 32 of Leprosy Review Vol. 45, Number 1. The manuscript stated "does occur". This has been printed as "does not occur". We apologize for the error. 